\title{
Le thème de la réalité dans les expositions d'art contemporain organisées par les musées de RFA dans les années $1970^{1}$
}

\author{
Mathilde Arnoux
}

La mimesis désigne le rapport d'imitation que l'art entretient avec la réalité. Il n'est pas question de sa restitution fidèle, mais de l'invention d'une représentation figurée dont le contenu est apprécié en fonction du lien qu'il entretient avec une extériorité visible ou invisible. Ces référents extérieurs les plus fidèlement rendus, ou bien transformés, magnifiés, déformés par l'imagination et les choix de l'artiste constituent la réalité de la représentation, à laquelle le spectateur doit croire. C'est du moins en ces termes que s'est posée la question de l'art jusqu'au XIX ${ }^{\mathrm{e}}$ siècle, opposant les catégories idéaliste, réaliste ou encore naturaliste, pour qualifier le degré de fidélité au monde extérieur reconnu aux représentations. Ce que la mimesis pose comme lien d'imitation entre l'art et la représentation du monde visible ou invisible, semble être devenu impropre pour apprécier les pratiques artistiques qui depuis la fin du XVIII ${ }^{\mathrm{e}}$ siècle n'ont plus mis l'amélioration ou l'imitation de la nature au cœur de la représentation, mais l'expression du point de vue de l'artiste. La compréhension de l'art abstrait telle qu'elle s'affirme en Europe occidentale dans les années d'après-guerre consacre cette tendance. Pourtant, à la fin des années 1950 et au début des années 1960, après que l'abstraction occidentale a laissé pour morte la figuration, celle-ci revient en force. Face à des pratiques artistiques qui affirment leur nouveauté en revendiquant leur ancrage dans la réalité, la critique et l'histoire de l'art, pour en saisir la singularité par rapport aux expressions mimétiques d'autrefois, s'intéressent à la façon dont ces nouvelles pratiques se situent par rapport à

1. Les recherches menées pour écrire cet article ont été rendues possibles grâce au projet ERCStarting Grant « $\grave{A}$ chacun son réel » et à toute son équipe. Je tiens ici à remercier particulièrement Maria Bremer, doctorante du projet responsable de la RFA, Clément Layet, philosophe post-doctorant du projet et Sira Luthardt qui ont été des interlocuteurs essentiels au fil des travaux de réflexion menés pour cet article. 
l'imitation et au réalisme. À travers ces thèmes, les débats cherchent à statuer sur la modernité et l'originalité de ces pratiques selon la façon dont elles sont présentées en continuité ou en rupture avec le cours de l'histoire de l'art. Ce débat se joue également sur la toile de fond de l'affrontement idéologique entre bloc de l'Ouest et bloc de l'Est, dont les modèles rivaux se situent de manière très distincte. Les pratiques artistiques contemporaines sont envisagées à l'Ouest du point de vue de leur nouveauté, tandis qu'à l'Est, dans les pays qui pratiquent le réalisme socialiste, la figuration dialogue souvent avec les pratiques du XIX ${ }^{\mathrm{e}}$ siècle, et la nouveauté n'est pas un but. Le lien avec un débat aussi traditionnel dans les arts plastiques que celui de la mimesis est donc envisagé différemment selon la famille philosophique et politique à laquelle appartiennent les critiques et historiens de l'art. Les voix qui se manifestent alors sont plurielles, souvent antagonistes, et doivent être prises en considération dans leur diversité. En RFA, dans les années 1960, tandis que différents mouvements artistiques invoquent, chacun à leur manière, réalité et réalisme $^{2}$, un débat autour de ces mêmes thèmes s'établit. Il prend forme dans le discours des critiques d'art notamment à travers les revues Kunstwerk, Tendenzen, mais aussi dans la presse quotidienne ${ }^{3}$, ou encore dans des colloques ${ }^{4}$. Le réalisme est également au centre des débats qui animent alors l'histoire de l'art nourrie aux théories de l'école de Francfort en RFA, et se donne à lire à partir de 1974 dans les pages de Kritische Berichte, organe du Ulmer Verein für Kunst- und Kulturwissenschaft ${ }^{5}$. C'est dans ce contexte de convergence d'intérêt de la production artistique, de la réflexion théorique et des débats en histoire de l'art pour la

2. Pour exemple l'exposition de Wolf Vostell à la galerie Schwarz de Milan en 1961, Cityrama 1a. Démonstration permanente réalistique : murs et décollages à Cologne trouvé et sélectionné par Vostell; l'exposition Leben mit Pop. Eine Demonstration für den kapitalistischen Realismus. Düsseldorf, Möbelhaus Berges organisée à Düsseldorf en 1963 par Gerhard Richter et Konrad Lueg; ou encore l'exposition Phänomene und Realitäten organisée à la galerie René Block à Berlin en 1965.

3. Voir à ce sujet Jost Hermand, «Die "wirkliche" Wirklichkeit. Zum Realismus-Streit in der westlichen Kunstkritik », in : Reinhold Grimm, Jost Hermand (éd.), Realismustheorien in Literatur, Malerei, Musik und Politik, Stuttgart, Kohlhammer, 1975, p. 118-137 ; Jutta Held, « New Left Art History and Fascism in Germany », in : Andrew Hemingway (éd.), Marxism and the History of Art. From William Morris to the New Left, Londres, Pluto Press, 2006, p. 196-212 ; Maria Bremer synthèses sur l'usage du terme de réel et réalité dans Das Kunstwerk, à paraître sur http://www.own-reality.org/.

4. Colloque « Bildende Kunst und Wirklichkeit» organisé par le Dr. G. Böhm à l'académie évangélique de Baden en 1969.

5. En 1968, le Ulmer Verein für Kunst-und Kulturwissenschaft a été fondé à l'occasion du Verband Deutscher Kunsthistoriker. Il propose un programme de réforme radical de l'histoire de l'art. Partant d'une critique des théories et méthodes prédominantes, ses membres imprégnés notamment des leçons théoriques de l'école de Francfort, entreprennent une critique de la discipline. Ils contestent la prétention de l'histoire de l'art à être une science objective et insistent sur les sources idéologiques auxquelles elle puise. Les textes qu'accueille sa revue Kritische Berichte s'intéressent à la pédagogie en histoire de l'art et présentent des champs d'analyse innovants comme l'histoire sociale de l'architecture, l'art durant le national socialisme, ou encore l'histoire de la photographie. La revue propose également des recherches historiographiques sur les historiens de l'art Frederick Antall, Max Raphael et Arnold Hauser et traite de l'Ecole de Francfort comme modèle fondamental. Les thèmes centraux de la recherche de cette jeune génération d'historiens de l'art sont le réalisme et le fascisme. Voir à ce sujet Andrew Hemingway, « New Left Art History's International », in : Andrew Hemingway (éd.), Marxism and the History of Art. From William Morris to the New Left, Londres, Pluto Press, 2006, p. 175-195; 
réalité, le réel et le réalisme que se tiennent plusieurs expositions d'art contemporain sur ces thèmes en $\mathrm{RFA}^{6}$. Pour éclairer les questions soulevées par ces expositions, il faut revenir sur les enjeux des débats autour des termes de Wirklichkeit et Realität ${ }^{7}$ dans les années 1960 et sur leur lien avec le développement de l'histoire de l'art du XIX $X^{\mathrm{e}}$ siècle.

\section{L'imitation, enjeu de la rupture entre les pratiques artistiques traditionnelles et modernes}

En 1969, le compte rendu de Günter Metken, paru à la suite du colloque à l'académie évangélique de Baden intitulé «Bildende Kunst und Wirklichkeit », sous la direction du Dr. G. Boehm dans Das Kunstwerk ${ }^{8}$, permet d'apprécier comment les critiques et historiens de l'art envisagent certains aspects du débat des années 1960.

Le colloque, dans sa vision rétrospective de la production artistique occidentale depuis le tournant des XIX ${ }^{\mathrm{e}}$ et $\mathrm{XX}^{\mathrm{e}}$ siècles, a globalement constaté l'éloignement de la réalité figurée dans les pratiques artistiques. Comme la question de la figurabilité («Abbildlichkeit »), remarque Günter Metken, n’a pas été traitée une seule fois,

Jutta Held, op. cit. ; Klaus Herding, 1968 : Kunst, Kunstgeschichte, Politik, Francfort-sur-le-Main, Anabas Verlag, 2008.

6. Nous nous concentrerons ici sur quelques expositions qui ont mis la réalité au centre de leur propos et sur ce que l'emploi du terme de réalité (Wirklichkeit ou Realität) soulève comme question à l'interprétation de la création artistique de ces années : Realität - Realismus - Realität, cat. exp. Wuppertal, Von der Heydt-Museum, 28.octobre 1972 au 17 décembre 1972; Realismus und Realität, Ausstellung zu 11. Darmstädter Gespräch veranstaltet von der Stadt Darmstadt zusammen mit dem Kunstverein Darmstadt, cat. exp. Darmstadt, Kunsthalle, 24 mai au 6 juillet 1975; Als guter Realist muß ich alles erfinden. Internationaler Realismus heute, cat. exp. Hambourg, Kunstverein und Kunsthaus Hambourg, 4 novembre 1978 au 7 janvier 1979. Il ne sera donc pas question d'une analyse des nombreux ouvrages sur le réalisme paru dans les années 1970 comme celui de Udo Kultermann, Radikaler Realismus, Tübingen, 1972, Peter Sager, Neue Formen des Realismus. Kunst zwischen Illusion und Wirklichkeit, Cologne, 1973, ni des expositions consacrées au réalisme : Prinzip Realismus. Malerei, Plastik, Graphik, au Badischer Kunstverein de Karlsruhe en 1974 ; D-Realismus. Realistische Kunst in der Bundesrepublik Deutschland, au Kunstverein de Kassel en 1977, ni même d'une histoire du réalisme comme la propose Boris Röhrl (Boris Röhrl, Realismus in der bildenden Kunst. Europa und Nordamerika 1830 bis 2000, Berlin, Gebrüder Mann Verlag, 2013). Nous ne traiterons pas ici des trois Documenta qui dans ces années ont mis la question de la réalité au centre de leur organisation : la 4 en 1968 à travers le Pop, la 5 en 1972 à travers le «Questionnement à la réalité » organisé par Harald Szeemann, et la 6 en 1977 autour du médium. La question de la réalité dans le concept et la réalisation de l'exposition de 1972 fait l'objet d'un article approfondi de Maria Bremer auquel nous renvoyons : «Documenta 5 - Konzept und Realisierung », ainsi que le concept de la Documenta 6 « Kein Einklang. Selbstreferenz, Realismus und Anthropologie im ,Medienkonzept' der documenta 6 » à paraître dans la série de publication Ownreality, http://www.own-reality.org/.

7. Les notions de réel et réalité (Wirklichkeit, Realität) sont fréquemment invoqués dans le discours sur l'art, par la critique comme par les artistes à partir de la fin des années 1960 en Europe occidentale, sans qu'il n'y ait de différenciation claire entre les deux termes.

8. Günter Metken, «Bildende Kunst und Wirklichkeit », Das Kunstwerk. Zeitschrift für moderne Kunst 7-8, 1969, p. 49. 
la notion de réalité des années 1960 a peu à voir, lui semble-t-il, avec celle du réalisme du XIX ${ }^{e}$ siècle pour lequel l'enjeu était, à ses yeux, d'utiliser ou de citer la réalité, alors que le $\mathrm{Xx}^{\mathrm{e}}$ siècle a pour ambition de la transformer. Metken pose ainsi d'emblée un changement profond du lien de l'art à la réalité au XX $\mathrm{XX}^{\mathrm{e}}$ siècle : il n'est plus question pour l'art d'imiter, de copier la nature, ni de se situer dans un rapport de dépendance de l'art au monde visible, il s'agit d'envisager la dimension performative de l'art, générateur de réalité par lui-même.

La même année, l'ouvrage de Werner Hofmann intitulé Von der Nachabmung zur Wirklichkeit. Die schöpferische Befreiung der Kunst 1890-19179 (De l'imitation à la réalité. La libération créatrice de l'art 1890-1917) pose lui aussi une rupture centrale qui éclaire celle que Metken a établie entre le réalisme et la réalité dans les années 1960. Hofmann y établit les origines de la crise de la peinture de chevalet au tournant des $\mathrm{XIX}^{\mathrm{e}}$ et $\mathrm{XX}^{\mathrm{e}}$ siècles, et, de là, instaure une opposition entre la période qui précède ce moment et celle qui lui succède ${ }^{10}$. Pour Hofmann, le tournant est représenté par le Jugendstil, Dada, De Stijl et le constructivisme pour lesquels la peinture de chevalet n'est plus le seul but de la peinture. Ces mouvements se livrent plutôt à des pratiques hybrides entre peinture et sculpture, sculpture et architecture, entre œuvre d'art et objet utile dont les œuvres Néo-Dada, nouveau réalisme, Pop Art, Op Art et l'ABC-Kunst proposent des prolongements. L'art n'a plus dès lors pour but l'imitation («Nachahmung »), mais sa réalité propre («Wirklichkeit»), que ce soit par exemple avec l'utilisation de matière brute, la présentation d'objets volontairement non artistiques ou l'affirmation de la réalité autonome des formes et couleurs constituant la peinture. Ainsi la réalité, c'est le monde extérieur qui pénètre dans l'œuvre à travers la matière, les objets qui ne sont plus des représentations, mais donnés tels quels pour ce qu'ils sont. La réalité, c'est aussi la peinture qui n'a pas d'autre sujet qu'elle-même. La réalité est un moyen pour Hofmann de poser la nouveauté du $\mathrm{XX}^{\mathrm{e}}$ siècle, son affranchissement à l'égard des débats autour de la mimesis : comme l'indique le titre de l'ouvrage, les siècles de l'imitation sont opposés au siècle de la réalité.

Les thématiques développées par Werner Hofmann dans son analyse du tournant des $\mathrm{XIX}^{\mathrm{e}}$ et $\mathrm{XX}^{\mathrm{e}}$ siècles entrent en résonnance avec les interprétations proposées au colloque de Baden de certains mouvements des avant-gardes historiques, mais aussi avec des pratiques contemporaines. Ainsi, on retrouve le thème d'un art envisagé dans son autonomie à travers les analyses de la théorie de l'art concret de Van Doesburg et Mondrian. Le colloque de Baden montre qu'en faisant des lois de la construction, le sujet et l'objet des œuvres, les artistes conçoivent la réalité en terme de contenus généraux (les Realien). Il n'est plus question du monde visible (« das Sichtbare »), ni d'un

9. Werner Hofmann, Von der Nachabmung zur Wirklichkeit. Die schöpferische Befreiung der Kunst 1890-1917, Cologne, DuMont, 1969.

10. La recherche de Werner Hofmann a pour point de départ la thèse de Clement Greenberg, dont Hofmann avait lu en 1948 l'article « The Crisis of the Easel Picture » paru dans le Partisan Review. Il explique dans l'introduction à Von der Nachabmung zur Wirklichkeit. Die schöpferische Befreiung der Kunst 1890-1917, avoir par la suite oublié le nom de l'auteur et le contenu du texte, mais pas son sujet qui a guidé ses interrogations et l'a conduit à entreprendre des recherches sur les origines de l'émancipation de la peinture de chevalet, dont il a publié les premières conclusions dans Grundlagen der modernen Kunst, Stuttgart, 1966. 
quelconque rapport mimétique de l'œuvre au monde extérieur. La réalité est aussi au cœur de la conférence sur le Happening. Il est présenté comme une critique du rapport élitiste à l'artefact, tel qu'envisagé par la société bourgeoise. De telles pratiques en se mettant à distance de la représentation visent à réduire la différence entre l'art et la vie. Mais cette tentative est présentée comme un échec : à vouloir échapper à l'art, le happening a fini par ne plus attirer que des étudiants rebelles sans véritablement parvenir à constituer un public. L'art de l'objet à travers le collage est analysé, quant à lui, comme une implication de la réalité dans l'art («Einbeziehung von Wirklichkeit in die Kunst »). Les lambeaux de réalité ( «Wirklichkeitsfetzen ») utilisés à des fins formelles par les cubistes, les dadaïstes ou les surréalistes véhiculent des réalités différentes selon les mouvements artistiques. Ce caractère central de la citation, du montage et de la transformation de morceaux de réalité («Wirklichkeitsteilen »), se retrouve dans les grandes lignes de l'ouvrage de Herta Wescher, Die Collage. Geschichte eines Ausdrucksmittel (Cologne, 1968) paru un an auparavant. Enfin, l'image soumise par l'œuvre Pop tend à faire coïncider l'art et la réalité ( Gleichsetzung der beiden, Wirklichkeit und Kunst ») de manière artificielle grâce à des media anonymes, proches du ready-made, de la télévision, de la publicité, de la bande dessinée. Il n'est plus question de représentation figurée par l'artiste, ce que celui-ci produit peut être confondus avec des produits de consommation.

Les points de vue développés dans le colloque de Baden sur le lien entre l'art et la réalité écartent la question de la mimesis envisagée de manière traditionnelle comme imitation du monde visible. La complexité des débats autour de la question de l'imitation de la nature, que ce soit à l'académie des Beaux-Arts, dans les écrits de Baumgarten sur la copie et à sa suite dans l'esthétique allemande, le Laocoon de Lessing, ou encore dans les controverses autour du réalisme en France au XIX ${ }^{e}$ siècle, n'est pas mentionnée. Le $\mathrm{XX}^{\mathrm{e}}$ siècle a fait place aux réalités caractéristiques des pratiques contemporaines, que les textes de Hofmann, de Herta Wescher sur le collage ou les débats menés à Baden cherchent à historiciser, en les inscrivant dans l'héritage des avant-gardes. Par l'établissement de réalités qui se démarquent de l'imitation, ces discours structurent l'histoire de l'art du XX siècle autour d'une rupture entre les pratiques d'avant-garde et celles qui se fondent sur l'imitation, la figuration du monde visible. Ils établissent ainsi des héritages différenciés entre les réalités des avant-gardes d'une part, et les imitations entendues comme traditionnelles de l'autre. À travers ce jeu d'opposition se dessine l'affrontement entre les pratiques contemporaines empruntant aux avant-gardes occidentales d'une part et le réalisme socialiste nourri aux figurations académiques d'autre part.

Il n'est cependant pas seulement question d'une bipolarisation entre imitation et réalités, ou entre XIX ${ }^{\mathrm{e}}$ et $\mathrm{XX}^{\mathrm{e}}$ siècles, ou finalement entre l'Est et l'Ouest. C'est aussi la supériorité de l'abstraction sur toute autre forme d'expression qui est en jeu, de cette abstraction investie par le bloc de l'Ouest comme manifestation par excellence de ses valeurs de liberté ${ }^{11}$. Or la revue Das Kunstwerk, qui relaie le débat du colloque de Baden, a depuis 1946 défendu la ligne de l'abstraction lyrique, à travers un intérêt particulier pour la position française moderne et l'abstraction américaine ${ }^{12}$.

11. Serge Guilbaut, Comment New York vola l'idée d'art moderne : expressionnisme abstrait, liberté et guerre froide, Paris, Hachette littérature, 2006.

12. Au sujet de la revue lire Maria Bremer, Das Kunstwerk, à paraître sur http://www.own-reality.org/. 
À la suite de Werner Haftmann, l'un des pères du discours sur l'art moderne d'après-guerre en Allemagne, l'abstraction est entendue notamment par les critiques de cette revue comme donnant accès à la réalité ${ }^{13}$. L'art n'est pas déterminé par une fonction, il est le lieu même de la réalité, dans lequel l'individu existe pour lui-même ${ }^{14}$. En se rattachant à l'héritage de la théorie esthétique romantique dans son opposition fondamentale à l'enseignement de la copie de la nature, Werner Haftmann fonde le sujet créateur autonome et le génie comme creuset de toute création artistique. Il attribue à l'abstraction la qualité de saisir la réalité par-delà les apparences, une réalité qui puise à la fois dans les expériences que l'humain fait du monde intérieur et du monde comme tout, dans lequel se situe la dimension universelle et cosmique de l'être. Das Kunstwerk a longtemps défendu cette ligne, et a d'abord tenu en mépris l'art de l'objet et le Pop Art. Mais ceux-ci s'imposant progressivement sur la scène contemporaine, les critiques les ont intégrés à leur réflexion ${ }^{15}$. Le colloque de Baden est exemplaire à ce titre. L'art de l'objet et le Pop y sont reconnus, mais restent néanmoins dépréciés par rapport à l'abstraction.

Ce colloque fait en effet apparaitre une hiérarchisation des pratiques artistiques au sein du discours. Il situe les abstractions à un plus haut degré de conceptualisation que l'art de l'objet, et présente le Pop comme allant de pair avec une désesthétisation et une dépersonnalisation propre à la réalité du bien de consommation. En raison de leur référence au monde environnant, ils sont moins estimés que l'abstraction. La réalité matérielle introduite dans l'œuvre est jugée triviale, prosaïque, vulgaire ${ }^{16}$. Ce mépris pour la réalité matérielle par comparaison avec la réalité intellectuelle et spirituelle sur laquelle ouvre l'abstraction, s'apparente aux résistances des idéalistes contre le réalisme. C'est la lecture d'un texte de Winfried Nerdinger paru quelques années plus tard qui nous invite à établir cette parenté. Ce critique y étudie le terme de réalisme en France et son application au domaine de l'art non figuratif ${ }^{17}$. Il présente une historiographie du réalisme, revenant aux débats

13. Sous la direction d'Arnold Bode, Werner Haftmann a été chargé d'élaboré la problématisation des trois premières documenta I (1955), II (1959) et III (1964) de Kassel qui ont contribué à établir son point de vue auprès du grand public. Il est également l'auteur d'un panorama de la peinture du XX $\mathrm{X}^{\mathrm{e}}$ siècle, qui se concentre sur les pratiques occidentales, et dont le succès conduit à de nombreuses rééditions, voir Werner Haftmann, Malerei im 20. Jabrbundert, Munich, Prestel Verlag, 1954, voir son introduction à l'édition de 1955. Au sujet de Werner Haftmann voir Harald Kimpel, «Werner Haftmann und der Geist der französischen Kunst», in : Martin Schieder et Isabelle Ewig (éd.), In Die Freibeit geworfen. Positionen zur Deutsch-französischen Kunstgeschichte nach 1945, Berlin, Akademie Verlag, 2006, p. 129-150 ; voir également Gregor Wedekind, «Abstraktion und Abendland », in : Nikola Doll et al. (éd.), Kunstgeschichte nach 1945. Kontinuität und Neubeginn in Deutschland, Cologne/Weimar, Böhlau Verlag, 2006, p. 165-181.

14. Voir Werner Haftmann, Malerei im 20. Jahrbundert, Munich, Prestel Verlag, 1954, voir son introduction à l'édition de 1955 , p. 31.

15. Jost Hermand, « Die "wirkliche" Wirklichkeit », op. cit.

16. Voir notamment à ce sujet le débat autour du Pop dans Das Kunstwerk, 1964, cahier 10, $\mathrm{n}^{\circ} 17$, mais également Jost Hermand, Pop international. Eine kritische Analyse, Francfort-sur-le-Main, Athenäum, 1971.

17. Winfried Nerdinger, «Zur Entstehung des Realismus-Begriffs in Frankreich und zu seiner Anwendung im Bereich der ungegenständlichen Kunst », Städel-Jabrbuch 5, 1975, (p. 227-246), p. 234 et suivantes. 
du XIX ${ }^{e}$ siècle et, en particulier, aux résistances des idéalistes au positivisme de Comte et au socialisme de Saint Simon. Ainsi les idéalistes y sont présentés comme ne voyant dans les paysages de la province française d'artistes comme Troyon, ou Cabat, qu'une reproduction purement matérielle, qu'ils comparent à des daguerréotypes peints. Le positivisme, défini par Comte dans sa Sommaire appréciation sur l'ensemble du positivisme de 1820 comme embrassant l'empirisme, la technique, la réalité quotidienne et l'industrie, représentait pour les idéalistes, rappelle Nerdinger, la couche la plus basse, la plus matérielle de la réalité. Or c'est bien en ces mêmes termes que les tenants de l'abstraction appréhendent la réalité dans le Pop. Les objets intégrés dans les œuvres sont présentés comme introduisant littéralement des éléments du monde environnant, et le Pop apparaît comme une reproduction d'images issues du monde des représentations quotidiennes. Le choix de ces objets au sein du monde environnant, leur transformation ou leur montage dans une composition mais aussi leur incidence sur le sens de ces œuvres ne sont ici nullement pris en compte. Même si ces jugements de valeur font écho au débat du XIX $X^{e}$ siècle entre idéalistes et réalistes, il n'est pas question pour autant de faire de ces pratiques contemporaines un réalisme. La réalité de l'art de l'objet ou du Pop est ainsi présentée dans un rapport quasi tautologique à la réalité qu'ils représentent. L'extériorité est transportée dans l'œuvre, sans médiation, sans transformation, en tant que telle. Ces tendances sont historicisées en les distinguant de tout rapport mimétique. La tautologie apparaît comme une marque de leur inscription dans l'héritage des avant-gardes pour éviter toute confusion avec les pratiques artistiques mimétiques, et particulièrement les réalismes socialistes.

Ces débats s'organisent donc autour d'une opposition entre abstraction et figuration, invention et imagination, réalité spirituelle et réalité matérielle, progressisme et obscurantisme. La question de la mimesis permet de se démarquer d'un héritage classique et du réalisme auquel s'oppose l'art contemporain occidental. La réalité apparaît comme un terme discriminant qui permet d'identifier, selon la façon dont il est envisagé, des postures différentes. Elles sont éclairées par un article de 1975, intitulé « Die "wirkliche" Wirklichkeit . Zum Realismus-Streit in der westlichen Kunstkritik », dans lequel Jost Hermand ${ }^{18}$, historien de la culture, contributeur aux Kritische Berichte, distingue trois attitudes possibles par rapport aux mouvements qui affirment la nouveauté de leur pratique à travers la revendication d'un lien à la réalité : 1) rester fidèle à l'abstraction et rejeter ce lien, 2) s'habituer à commercialiser le réalisme et à le dégrader progressivement dans le manège des -ismes de l'art, en le laissant apparaître comme un épisode de courte durée ou, 3) saluer le nouveau réalisme comme un tournant dans la critique sociale et l'encourager fortement. La première attitude, incarnée par les tenants de la Klassische Moderne, oppose la réalité moderne d'avant-garde au réalisme traditionnel repris par le socialisme, et établit au sein de la réalité une hiérarchie entre réalité matérielle et réalité spirituelle. La seconde a admis le capitalisme et s'enthousiasme avec la même conviction pour toute nouvelle mode. Ainsi, selon cette seconde attitude, tout est acceptable tant que l'intérêt est suscité et la mise sur le marché garantie. Enfin, la troisième, représentée par la gauche, intègre au terme de

18. Jost Hermand, «Die "wirkliche” Wirklichkeit », op. cit. 
réalisme, lui-même distingué de la tradition mimétique, toutes les manifestations d'un art qui affiche un lien à la réalité en cherchant à la transformer. Elle s'appuie sur les définitions du réalisme développées par des auteurs marxistes, notamment Brecht, et s'intéressent aux théories qui paraissent dans Bildende Kunst, revue d'art officielle de la RDA.

C'est dans ce contexte conflictuel imprégné d'idéologies que plusieurs expositions tentent au début des années 1970 d'explorer le thème de la réalité dans l'art contemporain. Ces expositions suivent-elles le discours de la critique selon la ligne de Jost Hermand ? Sont-elles organisées selon les catégories établies par la critique et l'histoire de l'art? Reproduisent-elles les termes du débat idéologique? Les œuvres qu'elles réunissent sont-elles mises au service d'un discours normatif ? La réalité abordée y est-elle aussi assimilée au réalisme?

La plupart de ces expositions attestent un désir d'éclaircissement des enjeux que soulève la question de la réalité. Chacune approfondit certains aspects du débat des années antérieures. La recherche de distinction entre réalité et réalisme se retrouve ainsi au cœur de deux expositions organisées l'une à Wuppertal en 1973 (Realität, Realismus, Realität), l'autre à Darmstadt en 1975 (Realismus + Realität). Les différenciations constituent un objet d'intérêt particulier au début des années 1970, mais elles ne sont pas interprétées de la même manière selon les auteurs.

\section{La réalité matérielle s'oppose au réalisme illusionniste}

Realität, Realismus, Realität ${ }^{19}$ prend le parti de distinguer la réalité introduite pour ce qu'elle est dans certaines œuvres, de la réalité objet de l'imitation, propre au réalisme, due à l'artiste, qu'il soit idéaliste ou réaliste. Ainsi pour Johann Heinrich Müller « toute activité représentative, qu'elle repose sur une conception idéaliste ou réaliste, est dans tous les cas une traduction et une sélection de ce qui préexiste par un sujet réfléchissant, une production "issue de l'esprit" (Liebermann) $»^{20}$. L'exposition est envisagée comme une «présentation de l'histoire du matérialisme dans les nouvelles formes d'art $»^{21}$ et a pour ambition de se concentrer sur des œuvres

19. L'exposition Realität, Realismus, Realität a d'abord été présentée au Van der Heydt Museum à Wuppertal sous la responsabilité de Günter Aust et Johann Heinrich Müller, puis à la Haus am Waldsee de Berlin (Thomas Kempas), à la Kunsthalle de Kiel (Jean Christian Jensen et Karlheinz Nowald), à la Kunsthalle de Bielefeld (Joachim Wolfgang von Moltke et Hans Georg Gmelin), au Wilhelm-Lehmbruck-Museum de Duisburg (Siegfried Salzmann et Tilmann Osterwold), au Westfälischer Kunstverein de Münster (Klaus Honnef) et au Städtisches Museum de Leverkusen (Rolf Wedewer).

20. « Alle bildnerische Tätigkeit, ob auf idealistischer oder realistischer Anschauung beruhend, ist in jedem Falle eine durch ein reflektierendes Subjekt bedingte Übersetzung und Selektion des Vorgefundenen, ein Produzieren "aus dem Kopf" (Liebermann) » dans Johann Heinrich Müller, « Realismus - Kunst gegen die Philosophen», in : Realität, Realismus, Realität, cat. exp. Van der Heydt Museum, Wuppertal, 1973, p. 3-36, p. 9.

21. «Darstellung der Geschichte des Materialismus in der neuen Kunst » dans l'introduction signée par les responsables de l'exposition pour chacun des musées, in : Realität, Realismus, Realität, op. cit. 
qui conservent des morceaux non manipulés de la réalitée ${ }^{22}$. Ces morceaux de réalité non manipulés ont été introduits de manière exemplaire et décisive par trois artistes : Marcel Duchamp, Andy Warhol et Joseph Beuys. Marcel Duchamp a ouvert le mouvement avec ses ready-made. Andy Warhol, quant à lui, a introduit le mécanisme de la consommation avec le plus de pertinence dans le domaine de l'art, mais aussi la seconde épaisseur de réalité dans la photographie et la reproduction (la réalité n'y étant pas seulement celle de l'objet représenté qui fait image, mais celle du medium et de ses incidences sur la reproduction), et enfin la réalité de la rationalité technique de la production industrielle ${ }^{23}$. Joseph Beuys convoque la réalité à travers les rites de conjuration magiques émotionnels et montre un art de l'objet aux qualités de reliques. Dans cette opposition ainsi conçue entre la réalité et le réalisme, il y aurait d'une part des pratiques comprenant des éléments de réalité non transformée et de l'autre la représentation, réaliste ou idéaliste, de toute façon illusionniste. Cette présentation avoisine celle de l'artiste en scientifique objectif tel que le donnait à voir l'exposition de 1968. L'œuvre est le lieu de manifestation de la réalité matérielle (Duchamp), économique et sociale (Warhol), psychique et spirituelle (Beuys). Elle s'inscrit ainsi dans ces mêmes champs pour en devenir un élément, à la suite d'opérations précisément détaillées dans le cas du travail d'Andy Warhol ${ }^{24}$. Cette incorporation de l'œuvre à la société établit un changement de fonction de l'art et du rapport que le spectateur entretient avec lui. L'art n'est pas posé comme l'invention d'une représentation, il ne participe pas d'un monde parallèle, il est la restitution fidèle du monde extérieur matériel, de ses manifestations sociales, politiques et économiques. Cet art renseigne, fait prendre conscience au spectateur de questions soulevées dans ces différents champs du monde extérieur. Il a une fonction pédagogique. Il révèle l'importance de sujets dont le spectateur n'a pas conscience, et selon la spécialité de l'artiste le renseigne sur des questions économique, sociale, psychique. Ce discours écarte ainsi la spécificité de l'invention dans ses combinaisons complexes qui constituent la réalité même de la représentation. Un tel art n'interroge pas la représentation, mais double en quelque sorte les sciences humaines et sociales.

\section{La réalité d'une œuvre n'est pas l'objectivité scientifique. Pour une « réalité esthétique »}

Une même volonté de distinguer la réalité du réalisme peut conduire à des conclusions opposées, comme le montre l'exposition Realismus + Realität ${ }^{25}$

22. Ibid., p. 3 : «Wir möchten in dieser Ausstellung die Geschichte des unmanipulierten, unverstellten Stücks Wirklichkeit in der Kunst in einigen Strängen und an Beispielen zeigen ».

23. Ibid., p. 3 : «Er hat am folgerichtigsten den Mechanimus des Konsums, die zweite Wirklichkeitsschicht im Foto und in der Reproduktion, schließlich die technische Rationalität industrieller Fertigung, als auf den Verbraucher zugeschnittene Aspekte der Realität in den Bereich der Kunst geholt oder Kunst dieser Wirklichkeiten ausgeliefert ».

24. Tilman Osterwold, «Realität/Warhol/Realismus », dans Realität, Realismus, Realität, op. cit., (p. 67-111), p. 104.

25. Realismus und Realität Ausstellung zum 11. Darmstädter Gespräch veranstaltet von der Stadt Darmstadt zusammen mit dem Kunstverein Darmstadt, cat. exp. Darmstadt, Kunsthalle, 24 mai au 6 juillet 1975. 
organisée à la Kunsthalle de Darmstadt en 1975. Elle rassemble les diverses tendances de l'entre-deux-guerres parmi lesquels se trouvent notamment des représentants de la Nouvelle objectivité, Duchamp, Dali, Chirico, Magritte, Schwitters, Bissier, Morandi, et pour la période contemporaine les tenants du Pop Art anglais et américain, Eduardo Arroyo, certains hyperréalistes américains, Wolfgang Gäfgen, Johannes Grützke, Renato Guttuso, Yves Klein, Gerhardt Richter, George Segal, Gérard Titus Carmel, Werner Tübke, parmi d'autres, comme pour réconcilier ou du moins rassembler toutes les pratiques artistiques qui font l'objet du débat autour de la réalité, du réel et du réalisme. Le texte de Heinz Winfried Sabais, maire de Darmstadt et écrivain, intitulé «Kunst und Realität », publié dans le catalogue de l'exposition, dégage un fil conducteur rassemblant toutes ces pratiques artistiques. Si l'artiste observe le monde pour le représenter, ce qu'il produit est toujours un artefact et l'on retrouve ici les arguments de Heinrich Müller, développés dans le catalogue de Wuppertal, au sujet de la dimension illusionniste des pratiques artistiques, qu'elles soient réalistes ou idéalistes. Sabais se distancie de l'interprétation de l'artiste comme scientifique, et précise que jamais l'artiste n'offre une analyse objective de la réalité. Pour Sabais, s'inscrivant dans l'héritage d'un Werner Haftmann, chaque artiste crée à travers son œuvre une « réalité esthétique » qui est réelle en soi. L'œuvre possède une «réalité esthétique » et constitue un monde parallèle à celui des objets et des sujets du monde matériel. À l'opposé de l'exposition de Wuppertal qui présente l'histoire du matérialisme de l'art au $\mathrm{XX}^{\mathrm{e}}$ siècle, Sabais affirme l'autonomie des pratiques artistiques, même lorsqu'elles puisent leurs sujets dans le monde visible. Le rejet de la réalité dans l'art comme objectivation va de pair avec un rejet du réalisme. Sabais l'identifie comme un moyen de catégoriser ou d'orienter le débat idéologiquement, en référence au réalisme socialiste. Les artistes réalistes du XIX ${ }^{\mathrm{e}}$ siècle ou de l'entre-deux-guerres ont, selon lui, introduit de nouveaux sujets dans la peinture, mais ne conduisent pas à une connaissance des réalités sociales et individuelles de leur temps. Les représentations réalistes socialistes ne donnent, quant à elles, aucun accès aux réalités sociales. Compte tenu de la réalité esthétique de l'œuvre, de l'impossibilité pour une œuvre d'inclure la réalité extérieure non transformée, matérielle, sociale ou politique, rien n'est plus absurde aux yeux de Sabais que d'interroger la fonction sociale de l'art. Sabais, en opposition à ce qu'il identifie comme le déterminisme marxiste, invite à prendre en considération les recherches des sciences naturelles et physiques qui, à travers la théorie quantique, ont remis en question les liens de causalité.

Notons que tous les discours analysés, qui tentent de saisir la manifestation de la réalité dans l'art, en l'opposant le plus souvent au réalisme, interprètent cette transformation du sujet de l'art comme la manifestation d'un changement. Situé au tournant des $\mathrm{XIX}^{\mathrm{e}}$ et $\mathrm{XX}^{\mathrm{e}}$ siècles par Hofmann, il se matérialise dans le passage de l'imitation à la réalité et conduit dans les textes sur le Pop à la transformation de l'acte artistique en objectivation. Pour Sabais, ce changement relève des découvertes scientifiques, qu'il met directement en rapport avec ce que l'abstraction a représenté comme libération de l'exigence de l'art à représenter l'humain. Il est impossible de revenir sur cet apport des sciences, et de rétablir des catégories héritées du $\mathrm{XIX}^{\mathrm{e}}$ siècle. Le réalisme ne peut être ravivé, car il n'est pas adapté aux réalités manifestes dans les auvres. Sabais veut engager une nouvelle définition des pratiques 
figuratives. S'appuyant sur le relativisme introduit par les sciences naturelles et physiques, la figuration, estime-t-il, ne peut plus être considérée comme la représentation du monde visible ou l'expression des mouvements humains et sociaux. Ses propositions s'arrêtent à ce point, pour donner lieu à une conclusion en forme d'aveu d'une foi : «L'art comme compte rendu de réalités esthétiques émergeant d'une irrationalité inépuisable se poursuit. Il ne peut en aller autrement $»^{26}$.

\section{Le réalisme n'est pas seulement un reflet : Als guter Realist muss ich alles erfinden... 1979}

Dernière exposition à laquelle nous nous consacrerons, Als guter Realist muss ich alles erfinden organisée à Hambourg en 1979, présente des œuvres figuratives de David Hockney, Andy Warhol, Jim Dine, Alex Colville, Gerhard Richter, des artistes berlinois de Grossgörchen : Ulrich Baehr, Wolfgang Petrick, Klaus Vogelgesang, des artistes de RDA Arno Rink, Volker Stelzmann, ou encore de Renato Guttuso. Les textes du catalogue enregistrent la complexité des débats qui se sont stratifiés au fil des années et offre, en outre, une étude sur le réalisme, une réflexion approfondie sur le rapport entre l'art et la réalité, tentant d'en saisir les liens de manière renouvelée. Partant du titre de l'exposition issue d'une citation d'Alex Colville qui insiste sur la dimension inventive des pratiques réalistes pour mieux mettre à distance l'idée que le réalisme équivaudrait à une restitution fidèle du monde extérieur, l'exposition propose une réflexion sur la réalité à l'œuvre dans le réalisme, sans opposer les deux termes comme nous l'avons souvent vu faire précédemment. Cette exposition et le catalogue ne traitent pas d'un reflet de la réalité («Widerspiegelung der Wirklichkeit »), ni de copie descriptive (« Abschilderung »), ni non plus d'images issues du monde du visible ( «Bildern aus der Welt des Sichtbaren »), mais des propositions de chaque imagination individuelle. Elle interroge le caractère problématique d'une réalité, qui en plus d'être constituée d'éléments issus du monde visible, tient aussi aux peurs, aux souhaits, aux rêves, et aux cauchemars.

Uwe W. Schneede, qui a conçu l'exposition avec Klaus Herding, ouvre le catalogue par un texte reprenant le titre de l'exposition ${ }^{27}$, il y pose d'emblée les diverses façons dont les pratiques réalistes entretiennent un lien à la réalité. Il rappelle ainsi que pour certains le réaliste par excellence est Marcel Duchamp, tandis que pour d'autres il s'agit plutôt de Carl Andre. Cette multiplicité interdit l'établissement d'une définition stricte, mais doit ouvrir sur des thèses et des propositions. Pour Schneede, une discussion sur le réalisme nécessite de prendre en considération les transformations incessantes du terme. Il propose d'envisager les œuvres réalistes comme celles qui

26. Hans Sabais, «Kunst und Realität », in : Realismus und Realität, op. cit., (p. VII-X), p. X : « Die Kunst als Rapport der aus unerschöpflichen Irrationalität aufsteigenden ästhetischen Realitäten setzt sich fort. Das muss sein ».

27. Uwe W. Schneede, «Als guter Realist muss ich alles erfinden », in : Als guter Realist muss ich alles erfinden, cat. exp. Hambourg, Kunsthalle, 1979, p. 6-7. 
mettent en place l'émotion subjective à travers les hommes, l'environnement, les contextes sociaux, l'histoire, d'une manière telle que les proportions de cette émotion et que les hommes, l'environnement, les contextes sociaux, l'histoire peuvent être, malgré la mise en œuvre subjective de moyens et de méthodes esthétiques, authentifiés par le spectateur au regard de la réalité, et agir sur elle. Ceci pourtant d'une manière tout à fait compliquée et non-immédiate, car les moyens et méthodes esthétiques ont la plupart du temps non pas un caractère purement utile, mais constituent la réalité dans l'image et exigent donc d'être décodés ${ }^{28}$.

Ces quelques lignes situent le propos de l'exposition aux antipodes des propositions de Sabais à l'exposition de Darmstadt : la façon dont Schneede entend le réalisme est ce contre quoi s'élevait Sabais. Une lecture fondée sur une analyse sociale de l'œuvre, constituée autour de la dialectique marxiste, ayant pour but d'agir sur le monde, et les citations de Brecht, Lukács, Georg Schmidt qui émaillent le propos de Schneede sont là pour le confirmer. Mais on est bien loin d'une lecture simplificatrice telle que la vise Sabais d'un réalisme comme illustration des réalités sociales. À la suite de Brecht, puis de Georg Schmidt, le réalisme est envisagé comme une méthode, qui engage à un lien critique, sceptique avec ce qui existe, tout en manifestant la subjectivité de l'auteur. Il n'est pas question d'en arriver à porter un regard résigné sur l'idéal perdu, inatteignable, ni à une réconciliation des contradictions actives au cœur de la réalité, mais, selon une logique dialectique, de donner à voir ces contradictions, de les rendre visible pour que celui qui regarde l'œuvre se forme l'œil aux changements qui adviennent dans la réalité. La spécificité de l'œuvre réaliste est de donner accès au général à travers le particulier. Le discours de Schneede n'établit pas une réalité contemporaine en opposition avec un réalisme passé. Le réalisme contemporain qui englobe tous les modes de figuration s'inscrit dans une continuité avec les temps passés. Selon Schneede, l'innovation n'est pas le problème premier du réalisme, car il entretient souvent des liens étroits avec l'art des périodes antérieures. Schneede évoque le recours à la métaphore, aux juxtapositions, à l'allégorie par plusieurs artistes présentés dans l'exposition. Le point de vue sur la réalité qu'offre une œuvre réaliste est toujours, affirme Scheede, subjectif. On retrouve ici les caractéristiques du discours de gauche sur le réalisme, tel qu'il est décrit par Jost Hermand dans son article «Die "wirkliche" Wirklichkeit. Zum Realismus-Streit in der westlichen Kunstkritik $»^{29}$.

Il y a dans ces débats autour des questions de réalité, réel et réalisme dans les pratiques artistiques contemporaines des correspondances avec les écrits contemporains d'histoire de l'art sur le XIX ${ }^{\mathrm{e}}$ siècle. Nous avons vu comment le colloque de Baden faisait écho aux écrits de Werner Hofmann, comment l'interprétation du

28. Ibid., p. 6-7 : «Vielleicht sind realistische Kunstwerke solche, die subjektive Betroffenheit durch Menschen, Umwelt, gesellschaftliche Zusammenhänge, Geschichte so umsetzen, dass das Maß an Betroffenheit und dass die Menschen, die Umwelt, die gesellschaftlichen Zusammenhängen, die Geschichte vom Betrachter trotz des subjektiven Einsatzes ästhetischer Mittel und Methoden an der Wirklichkeit überprüft werden und auf diese Wirklichkeit einwirken können, dies jedoch in einer durchaus komplizierten und nicht unmittelbaren Weise, denn die ästhetischen Mittel und Methoden haben zumeist keinen bloß dienenden Charakter, sondern sie konstituieren Wirklichkeit im Bild, wollen also entschlüsselt sein ».

29. Voir Jost Hermand, « Die "wirkliche” Wirklichkeit », op. cit.. 
Pop pouvait être éclairée par la querelle entre positiviste et idéaliste telle qu'elle a été analysée par Winfried Nerdinger. Dans le catalogue d'exposition de Hambourg, ce dialogue est affirmé par la publication d'un texte de l'historien de l'art Klaus Herding, spécialiste de Courbet et du réalisme ${ }^{30}$, intitulé « Realismus. Eine Frage des Ziels $»^{31}$. Cette contribution s'inscrit dans une série de textes publiés par le même auteur et dont l'ambition est de cerner le réalisme ${ }^{32}$. Herding ouvre son article en constatant les nombreuses déclinaisons du terme de réalisme et s'interroge sur la pertinence à en faire encore usage. Il repère une tendance artistique qui ne se satisfait pas facilement de la réalité, qui se fixe pour objectif de déchirer le voile de la fiction et qui constitue le point de départ de sa réflexion sur le réalisme contemporain $^{33}$. Il dit s'inscrire dans la lignée de Jost Hermand qui dans son texte wirkliche Wirklichkeit tentait déjà d'établir une définition de la réalité. Il s'appuie sur les recherches de Winfried Nerdinger qui avait quelques années auparavant analysé les transformations du terme de réalisme au fil du temps et ses applications aux œuvres non figuratives ${ }^{34}$. Il se nourrit avant tout de ses propres recherches sur Courbet pour mener une analyse qui remonte à l'histoire du terme réalisme au XVIII et doit permettre d'en proposer une définition contemporaine. Se référant à Castagnary, Herding insiste sur le fait que dans le réalisme, il n'est pas question de fidélité absolue au détail, mais qu'il tient à une capacité de cerner la réalité, de l'encoder sans que les moyens soient nécessairement absolument figuratifs. La théorie de Brecht n'est pas adaptée selon lui à l'appauvrissement émotionnel, ni à l'endormissement politique auquel fait aujourd'hui face l'individu dans un Etat comme la RFA. C'est pourquoi il se réclame de «l'unidimensionnalité » de Marcuse. La théorie développée dans L'bomme unidimensionnel en 1964, puis dans La Dimension esthétique en 1977 établit l'impossibilité de l'utopie et de l'art qui l'incarne. Herding plaide en faveur d'un art qui ne réifie pas les liens socio-politico-culturels, puisque c'est, selon lui, en outrepassant les réalités établies que peuvent naître des missions inédites pour un art à nouveau réaliste. Herding clôt son texte en définissant le réalisme comme une question de but :

Le réalisme se définit à partir de son but. Les utopies concrètes, quant à elles, sont des denrées rares. C'est pourquoi une nouvelle définition de l'art réaliste doit être précédée par une définition du but de l'agir réaliste. Il est évident que seules des tendances qui définissent les faits [...] de manière « exacte » et les laissent en même temps ouverts à

30. Klaus Herding, « Traits de lumière d'Outre-rhin jetés sur la réception de l'artiste », in : Mathilde Arnoux et al. (éd.), Courbet à neuf !, Paris, MSH, 2010, p. 209-216.

31. Klaus Herding, « Realismus. Eine Frage des Ziels », dans Als Guter Realist muss ich alles erfinden, 1978, p. 12-13.

32. Voir aussi notamment Klaus Herding, « Mimesis und Innovation. Überlegungen zum Begriff des Realismus in der bildenden Kunst », Zeichen und Realität. Akten des 3. Semiotischen Kolloquiums Hamburg, Klaus Oehler (éd.), 2 t., Hambourg, Stauffenburg Verlag, 1981, p. 83-113 ; Klaus Herding, "Realismus", dans Kunst, Werner Busch et Peter Schmoock (éd.), 1987, p. 674-713.

33. Klaus Herding, « Realismus. Eine Frage des Ziels », op. cit., p. 12-13 : «Andererseits verweist dieser Begriff trotz seiner heutigen Unverbindlichkeit auf ein Bedürfnis, sich mit der Realität oder den Realitäten nicht einfach abzufinden, sondern sich mit ihnen auseinanderzusetzen, einen davor gezogenen Schleier von Fiktionen zu zerreißen ».

34. Winfried Nerdinger, op. cit. 
l'émotion, qui nomment les passions et les adversaires, qui saisissent les conditions sociales concrètes et qui imaginent des alternatives, seules ces tendances peuvent satisfaire un tel but - il s'agit là d'une perspective d'ensemble, non d'une exigence à l'égard de chaque œuvre d'art. Ce n'est qu'à ces conditions que le sauvetage du terme de réalisme deviendrait une chose réaliste ${ }^{35}$.

Les questions soulevées par la réalité font dans ce catalogue, comme dans le texte de Hermand, l'objet d'une recherche de définition d'un réalisme élargi.

\section{Conclusion}

Le réel et la réalité sont des termes dont l'usage permet d'identifier les familles intellectuelles auxquelles appartiennent les critiques et historiens de l'art des années 1960 et 1970. Les réalités ne sont pas toutes assimilées au réalisme. Elles sont entendues de diverses manières. Elles peuvent désigner une objectivité contemporaine, qui se distingue du régime d'invention et d'imitation antérieur après un moment de rupture. Elles se divisent aussi entre réalité matérielle des objets, des choses et réalité immatérielle englobant les expressions psychiques, émotionnelles, spirituelles. Elles sont également l'objet du réalisme entendu d'un point de vue marxiste, qui permet d'exposer par des artifices la réalité des aliénations, de la lutte des classes, de la dialectique. Qu'elle soit lue de manière progressiste et évolutionniste ou qu'on cherche au contraire à l'établir dans la tradition, la réalité fait débat. Elle oppose les auteurs sur des fronts souvent radicalisés entre posture idéaliste et posture réaliste, répétant les oppositions idéologiques et politiques de l'époque entre conservateurs et progressistes, entre blocs de l'Ouest et de l'Est. La réactivation du débat dans ses formes héritées du XIX ${ }^{\mathrm{e}}$ siècle ne peut être entendue comme le seul symptôme de positionnements idéologiques antagonistes. Cet intérêt pour le XIX $\mathrm{X}^{\mathrm{e}}$ siècle traduit également le besoin d'historiciser la question de la réalité dans l'art pour faire face aux interrogations que soulèvent les œuvres contemporaines. Car tous ces discours, quelle qu'en soit l'orientation, constatent d'importants changements, des transformations dans les liens de l'art à la réalité. Ces intuitions donnent lieu à des études antagonistes, issues de traditions analytiques différentes, sans que ne soit approché plus précisément le nouveau lien entre l'art et la réalité. Les discours établissent des démarcations nettes entre peinture, sculpture d'une part et pratiques conceptuelles, actions et happenings d'autre part dont les liens avec la réalité sont pourtant loin de s'opposer radicalement à ceux qui sont développées dans les pratiques traditionnelles. Dans un contexte qui expose la question des transformations du lien de l'art

35. Klaus Herding, « Realismus. Eine Frage des Ziels », op. cit., p. 21 : « Realismus definiert sich vom Ziel her. Konkrete Utopien aber sind Mangelware. Daher geht einer neuen Begriffsbestimmung realistischer Kunst die Zielbestimmung realistischen Handelns voraus. Dass einem solchen Ziel jedenfalls nur Richtungen genügen können, die Sachverhalte [...] - "exakt" definieren und zugleich emotional offenhalten, Leidenschaften und Gegner benennen, konkrete soziale Verhältnisse aufgreifen und Alternativen ersinnen, liegt auf der Hand - eine Gesamtperspektive, keine Forderung an jedes einzelne Kunstwerk. Erst unter dieser Prämisse wäre die Rettung des Realismusbegriffs eine realistische Sache ». 
et de la réalité, et en ouvre la définition par les réflexions qu'induisent de nouvelles formes de pratiques, il est intéressant de noter que la théorie de l'art s'intéresse alors aux travaux de Konrad Fiedler qui, un siècle auparavant, avaient invité à considérer l'œuvre non pas comme imitation servile, ou invention gratuite, mais comme le développement d'une réalité par l'artiste ${ }^{36}$. Les apports de la théorie de l'art ${ }^{37}$ exhortent l'histoire de l'art à remettre en question les catégories qui lui servent à traiter des pratiques qui convoquent la réalité, et à revisiter son écriture de la période de la guerre froide. L'historiographie de l'art du XIX ${ }^{\mathrm{e}}$ siècle a montré comment l'écriture de l'histoire de l'art avait participé à l'écriture des histoires nationales $^{38}$ et a ainsi pu étudier la perpétuation de ces positions analytiques et les limites de la discipline. L'intérêt porté aux années de guerre froide doit prendre en considération les positionnements idéologiques de l'histoire de l'art. Ils ont nourri des catégories et une vision bipolaire de l'art qui se perpétue encore aujourd'hui et empêche un regard renouvelé sur cette période, qui permettrait d'embrasser tout en les différenciant les divisions changeantes d'autrefois.

36. Voir à ce sujet Philippe Junod, «Actualité de Fiedler », Revue d'estbétique 26, 1973, p. 19-28 qui présente l'édition par Gottfried Böhm de Konrad Fiedler, Schriften zur Kunst, Munich, Fink, 1971. Voir également Konrad Fiedler, Sur l'origine de l'activité artistique, postface Danièle Cohn, Paris, éditions rue d'Ulm, 2008.

37. Au sujet des méthodes pour aborder la réalité en jeu des pratiques du XX⿳⺈冂大 siècle voir notamment Georges Didi-Huberman, Devant le temps. Histoire de l'art et anachronismes des images, Paris, éditions de Minuit, 2000 ; Catherine Perret, Les porteurs d'ombre. Mimesis et modernité, Paris, Belin, 2001.

38. Voir notamment Roland Recht et al. (éd.), Histoire de l'bistoire de l'art en France au XIXe siècle, Paris, La Documentation française, 2008 ; Hubert Locher, Kunstgeschichte als bistorische Theorie der Kunst 1750-1950, Munich, Fink, 2010 ; Michela Passini, La Fabrique de l'art national. Le nationalisme et les origines de l'bistoire de l'art en France et en Allemagne 1870-1933, Paris, MSH, 2012. 